\title{
Widespread imprinting of transposable elements and young genes in the maize endosperm
}

\author{
Sarah N Anderson ${ }^{1 *}$, Peng Zhou ${ }^{2}$, Kaitlin Higgins ${ }^{1}$, Yaniv Brandvain ${ }^{2}$, Nathan M Springer ${ }^{2}$ \\ ${ }^{1}$ Department of Genetics, Development, and Cell Biology; lowa State University; Ames, IA \\ ${ }^{2}$ Department of Plant and Microbial Biology; University of Minnesota; St. Paul, MN \\ * Corresponding author: Sarah N Anderson (sna@iastate.edu)
}

ORCIDs: 0000-0002-1671-2286 (SNA); 0000-0001-5684-2256 (PZ); 0000-0001-7759-4386

(KH); 0000-0002-3392-0220 (YB); 0000-0002-7301-4759 (NMS)

\begin{abstract}
Fertilization and seed development is a critical time in the plant life cycle, and coordinated development of the embryo and endosperm are required to produce a viable seed. In the endosperm, some genes show imprinted expression where transcripts are derived primarily from one parental genome. Imprinted gene expression has been observed across many flowering plant species, though only a small proportion of genes are imprinted. Understanding the rate of turnover for gain or loss of imprinted expression has been complicated by the reliance on single nucleotide polymorphisms between alleles to enable testing for imprinting. Here, we develop a method to use whole genome assemblies of multiple genotypes to assess for imprinting of both shared and variable portions of the genome using data from reciprocal crosses. This reveals widespread maternal expression of genes and transposable elements with presence-absence variation within maize and across species. Most maternally expressed features are expressed primarily in the endosperm, suggesting that maternal de-repression in the central cell facilitates expression. Furthermore, maternally expressed TEs are enriched for maternal expression of the nearest gene. Read alignments over maternal TE-gene pairs indicate fused transcripts, suggesting that variable TEs contribute imprinted expression of nearby genes.
\end{abstract}




\section{Main Text}

34 Imprinted genes showing parent-of-origin based patterns of expression were first identified in maize $^{1}$ and have since been identified in a variety of flowering plants. In plants, imprinted expression is primarily observed in the endosperm, which is a nutritive tissue of the seed that is formed when the diploid central cell is fertilized by one of the two sperm cells delivered by the pollen tube. The central cell is epigenetically distinct from most vegetative cells in the plant due to DNA demethylation targeted primarily to Transposable Elements (TEs) ${ }^{7-9}$. This demethylation acts as a primary imprint that distinguishes the female and the male alleles in the endosperm. Maternal and paternal alleles are further distinguished through differential accumulation of histone modifications such as $\mathrm{H} 3 \mathrm{~K} 27 \mathrm{me} 3^{10,11}$ which often marks the maternal allele of paternally expressed genes (PEGs) while maternally expressed genes (MEGs) often show differences in DNA methylation alone ${ }^{12}$. with conserved imprinting across species contribute to the establishment of imprinting ${ }^{13}$, several studies have observed substantial turnover of imprinting for many genes, either within a single species or across species ${ }^{14,15}$. However, understanding the rate of turnover and the source of the imprinted expression pattern has been challenging due in part to methodological

51 inconsistencies across studies and the limitations of available SNPs for allele calls. In

52 Arabidopsis, applying consistent methods and cutoffs across studies reduces apparent

53 variability in imprinting calls ${ }^{16,17}$, however many genes cannot be assessed due to a lack of

54 informative SNPs. A lack of SNPs can be due to identical sequence or unalignable regions

55 resulting from large structural changes or presence-absence variation (PAV) of whole genes or

56 features. In maize, many genes and TEs exhibit PAV among genotypes ${ }^{18-20}$. This limits the

57 ability to use SNP-based allele-specific expression analyses to study imprinting, especially for

58 transposons and variable genes. In this study, we develop an alternative approach that relies 
upon comparisons of expression in reciprocal crosses to assess the imprinting of both

conserved and variable genes and TEs across maize genotypes with whole genome

assemblies, revealing imprinting for many transposable elements and variable genic sequences.

Reciprocal crosses for every pairwise contrast between three maize genotypes with whole pollination, endosperm was isolated in triplicate for RNA-sequencing (Table S1). Two (SNP-ASE) followed by comparison of biases across reciprocal crosses (methods). The SNPASE ratio is calculated by assigning SNP-containing reads to one allele and determining the proportion of informative reads from each allele, providing an estimate of the expression of two

71 alleles within a single sample. We developed and implemented an alternative approach where reads are aligned to concatenated genome files and the Reciprocal Expression Ratio (RER) was calculated to describe the ratio of expression for features in each genome when inherited maternally versus paternally. Unlike SNP-ASE, the RER is a comparison of expression of a feature in reciprocal crosses and cannot be calculated for a single sample. Calculations of RER rely on the $\sim 15 \%$ of reads that map uniquely to a single location in the concatenated genomes (Table S1). While many reads map equally well to both genomes and are therefore discarded, unique mapping reads are only found in places of the genome with variants distinguishing the alleles (SNPs or indels) or in regions unique to one genome. After assigning unique reads to

80 features including genes and TEs using HTseq, RER was calculated by dividing the expression 81 level (RPM) when inherited maternally by the sum of expression when maternally or paternally

82 inherited. Given that endosperm is composed of two copies of the maternal genome and one 83 copy of the paternal genome, the null expectation for a transcript's expression is that it will be twice as highly expressed when inherited from the maternal parent compared to the paternal 
parent. For both SNP-ASE and RER, the average value representing a biparentally expressed gene is 0.67 , allowing direct comparison of the methods. A comparison of SNP-ASE and RER reveals general agreement between these two approaches for genes that could be analyzed with SNPs, with the majority of genes expressed at the ratio expected by dosage (Figure 1B). Many of the genes showing disagreement between methods in Figure 1B result from genotypebiased expression which exhibits a strong bias in SNP-ASE for a single sample but doesn't result in bias for RER (Figure S1). To further assess accuracy of RER, expression patterns for three MEGs and three PEGs with conserved imprinting status in maize, rice, and Arabidopsis ${ }^{4}$ were assessed (Figure 1C, Table S2). In most cases with informative reads, clear parental bias in the expected direction was observed for all genes (Figure 1C).

While both methods can be used to define imprinting for shared genes distinguishable by SNPs, only the RER method can capture imprinting for portions of the genome that exhibit PAV. This provides new opportunities to study parent-of-origin biased gene expression for TEs and variable genes. The distribution of RER values was assessed across contrasts for different feature types (Figure S2), and the proportion of each set that showed parentally-biased expression was summarized based on RER (Figure 1D). This revealed that across all contrasts, genes conserved within maize rarely exhibit parent-of-origin biased expression (Figure 1D, Figure S2). On average, $<3 \%$ of expressed genes that are present in all three maize genotypes in this study show a strong parental bias (Figure 1D). For genes that are variable among maize lines, a higher proportion (> 6\%) of expressed genes show high parental bias, with this set representing genes that are accessible using RER but not SNP-ASE. Strikingly, $>11 \%$ of expressed TEs show a strong parental bias, with the majority of strongly biased TEs expressed maternally (Figure 1D). 
110 In order to identify imprinted transcripts, we applied the IfcThreshold option within DESeq2 to

111 test for significance (adjusted p-value < 0.05) over the expected 2:1 gene dosage across

112 reciprocals using three biological replicates. To increase the stringency of imprinting calls,

113 significant hits were further filtered by RER values. Maternally Expressed Genes (MEGs) and

114 Maternally Expressed TEs (matTEs) were filtered for RER > 0.9, while Paternally Expressed

115 Genes (PEGs) were filtered for RER < 0.1. It can be difficult to remove all maternal tissues

116 when isolating endosperm tissue and therefore it is important to limit potential false-positive

117 calls of maternal expression that may result from genes expressed in the maternal seed coat ${ }^{24}$.

118 Previously published RNA-seq data ${ }^{25}$ was used to filter out genes whose maternal expression

119 could result from seed coat contamination rather than maternal expression in the endosperm.

120 Pericarp-preferred genes were defined where the mean expression in pericarp was >2-fold

121 higher than the expression in endosperm (Figure S3). After implementing these criteria and

122 filters, we identified an average of 182 total imprinted genes across all hybrid combinations, with

123 an average of $112 \mathrm{MEGs}$ and 70 PEGs in each (Figure 2A).

125 The imprinted genes discovered in each genome were compared to assess the consistency of 126 imprinting. A comparison of imprinted features in the B73 $\times$ W22 reciprocal hybrid endosperm 127 tissue identifies 17 MEGs, 39 PEGs, and 4 matTEs that were consistently imprinted in both 128 genomes (Figure 2B, 3B). A subset of the genes that do not exhibit consistent imprinting are 129 shared between the two genomes. For example, there are 26 MEGs observed only in B73 and 13011 only observed in W22 despite the fact that both genomes retain a syntenic ortholog for these 131 genes. For the majority of these shared genes with variable imprinting, the lack of overlap is due 132 to cutoff stringency or lack of coverage rather than true turnover of imprinting (Figure S4). There 133 are many additional cases where imprinted genes are only present in one genome. For PEGs, 134 variable genes represent the minority of non-conserved imprinted genes, with only 13 of 34 B73 135 PEGs that are not imprinted in W22 variable across genomes. In contrast, for the majority of 
MEGs with inconsistent imprinting (i.e. 107 of 133 B73 genes in the B73 by W22 contrast), the

137 genes themselves are absent from the other genome. Similar patterns are observed for the B73

138 by $\mathrm{PH} 207$ contrast, though a higher proportion of genes are shared in this contrast. The large

139 number of maternally expressed transcripts with variability in maize suggests that imprinting of

140 non-conserved elements may be far more prevalent than previously detected due to the

141 limitations of SNP-based allele calls.

143 To understand additional features of imprinted genes, we focused on the B73 genes that were called imprinted in at least one contrast, which included 202 MEGs and 111 PEGs. B73 was selected as the central genotype because it has substantially more expression datasets, syntenic gene information, and functional gene annotations than other genomes. For the genes identified as imprinted, we compared several characteristics relative to genes that were expressed but were not classified as imprinted. First, genes were assessed for variability across maize inbred lines by defining conserved genes as those with syntenic orthologs in B73, W22, and $\mathrm{PH} 207$ and variable genes as those without a corresponding gene in at least one genome

26. This revealed a clear enrichment for variable genes among MEGs ( $p$-value $<0.001$, chisq be assessed for imprinting (Figure 2C). We then expanded our evolutionary distance and assessed how many genes in each set are syntenic with other grasses as defined by having a syntenic ortholog in sorghum, rice, foxtail millet, and brachypodium. For genes without imprinting, the majority (62\%) are syntenic with other grasses. However, MEGs are highly

157 depleted for syntenic genes (19\%) and PEGs show a minor depletion (50\%, p-value $<0.05$,

158 chisq test). Next, the expression pattern across B73 development was assessed using 159 published RNA-seq data ${ }^{25}$. Since imprinting can arise from either silencing of one parental 160 allele specifically in the endosperm or de-repression of one parental allele in the endosperm, the 161 pattern of expression across tissues was defined as either constitutive or endosperm-preferred 
162 (see methods, figure S4). While only $3 \%$ of non-imprinted genes are expressed preferentially in 163 the endosperm, $77 \%$ of MEGs and $32 \%$ of PEGs show this expression pattern (Figure $2 \mathrm{C}, \mathrm{p}$ 164 value $<0.001$, chisq test). Many of the MEGs (38\%) have no assigned GO term, a 2.8-fold 165 enrichment compared to genes that are not imprinted ( $p$-value $<0.001$, chisq test). Since TEs 166 are a common source of new genes and a driver of gene content variation among maize lines, 167 we intersected our imprinted genes with annotated TEs, identifying 26 MEGs and 1 PEG completely within an annotated transposable element. While MEGs and PEGs are annotated as genes in the B73v4 annotation, transcription of a locus does not imply the creation of a functional gene product. While evolutionarily conserved genes with synteny to other grasses may be the best candidates for real genes capable of conferring phenotypes ${ }^{27}$, variable genes can be important for functions such as disease resistance ${ }^{28}$.

174 To further investigate the imprinting of TEs themselves, the RER method was used to define imprinted TEs, with an average of 95 matTEs identified across contrasts (Figure 3A). There are a small number of paternally expressed TEs, however these were excluded from further

177 analyses due to the low number detected and potential technical complications (Figure 3A, S2).

178 Consistent with the large amount of TE variability among genotypes, the majority of imprinted 179 TEs were unique to one genome (Figure 3B). There are 145 maternally expressed TEs in B73 180 relative to at least one other genotype, including 72 LTR retrotransposons, 52 Helitrons, 9 TIR 181 transposons, and 2 LINEs (Figure 3C). The vast majority of these TEs (93\%) represent specific 182 TE insertions that are polymorphic among the three maize genotypes ${ }^{20}$. Given the high tissue183 specificity of TE expression observed previously ${ }^{29}$, the tissue-specific expression patterns for 184 matTEs were also assessed. We found that $92 \%$ of matTEs are expressed preferentially in the 185 endosperm, suggesting that imprinting is established through de-repression of the maternal 186 allele preferentially in the endosperm and that this is the only stage of development for 187 expression of these elements (Figure 3C, S5). Since TE families have the potential for 
coordinated expression responses among members, the families for matTEs were assessed.

matTEs are in 84 families, with only one Helitron family containing more than 5 imprinted

elements. This family, DHH00002 (DHH2), contains 44 maternally expressed members and is

191 the only Helitron family in B73 that is predicted to have autonomous members. Since prior work

192 has suggested that Helitrons are responsible for creating imprinting by moving PHE1 binding

193 sites around the genome ${ }^{30}$, the proportion of $\mathrm{DHH} 2$ Helitrons with predicted motifs was

194 assessed (Figure S6). We found that matTEs of this family are more likely to have a binding site

than elements that are not detected in our analysis, though the distribution is similar to family

members that are not imprinted so it is unlikely that PHE1 sites alone are sufficient to confer imprinting of DHH2 Helitrons.

TEs have been proposed as a source of variation in imprinted gene expression, and this dataset nearest gene is a MEG, which is a significant enrichment ( $p$-value $<0.001$, binomial test) and 11.6 times more common than expected based on the proportion of expressed genes that are called MEGs (Figure 3D). In contrast, there were no identified examples of matTEs where the closest gene is a PEG. There were 19 matTEs where the closest gene is one of $15 \mathrm{MEGs}$ (Table S3). In the majority of cases, the TE overlapped $(\mathrm{N}=7)$ or was upstream of the gene ( $\mathrm{N}$ $207=10)$. We identified only two cases of the TE located downstream of the gene, and one of these 208 genes also overlapped a matTE. The asymmetry between upstream and downstream TE 209 relationships suggests that the orientation likely matters for determining which TEs are able to 210 influence gene expression patterning. In all cases, the developmental expression patterns of the 211 genes and the nearby TEs match. To understand the nature of transcripts, read alignments for 212 matTE-MEG pairs were visualized with IGV. In all cases, reads aligning to both the matTE and 213 corresponding MEG mapped to the same strand without clear separation in read alignments, 
214 suggesting that many of these clusters may actually represent single transcripts overlapping

215 multiple features (Figure 3E).

216

217 In summary, we developed the RER method to use information from shared and variable

218 portions of maize whole genome assemblies to identify imprinted expression of genes and TEs

219 in maize. This revealed imprinting of many genes that were undetectable by traditional methods

220 that rely on diagnostic SNPs between parental alleles. The majority of maternally expressed

221 features (genes and TEs) represent young portions of the genome that are variable within maize

222 and non-syntenic with other grasses. We also observe strong enrichment for MEGs near

223 maternally expressed TEs, further supporting the connection between turnover of imprinting and

224 presence-absence variation of TEs. In mammals, imprinting in the placenta has been proposed

225 to result from different defense mechanisms used by male and female germlines to reduce

226 retrovirus proliferation in the germ line ${ }^{31}$, and turnover of imprinting could have a similar host

227 defense explanation in plant endosperm. In plants, there are genes with conserved imprinting

228 across plant species that support theories of parental conflict ${ }^{32}$ or dosage ${ }^{33}$, however the

229 majority of imprinted loci are variable within and across species. By studying imprinting using

230 whole genome assemblies, we are able to better understand the turnover of imprinted

231 expression of both shared and variable portions of plant genomes. 


\section{Materials and Methods}

\section{Materials}

235 Three maize inbred lines, B73, W22, and PH207, were grown in the field in Saint Paul, MN in

236 the summer of 2018. Reciprocal crosses between each pair of genotypes were performed. Ears

237 were collected 14 days after pollination and endosperm was isolated using manual dissection,

238 with approximately 10 kernels per ear pooled for each biological replicate. Paired-end, stranded

239 RNA-seq libraries were created using the Illumina TruSeq Stranded mRNA kit and sequencing

240 was performed with the Illumina HiSeq 2500 at the University of Minnesota Genomics Center.

241 On average, > 45 million reads were generated per library (Table S1).

\section{Sequence alignments for RER}

244 Concatenated genome files were created for each pairwise contrast of parental genomes and assemblies used included B73v4 ${ }^{21}, \mathrm{~W} 22^{22}$, and $\mathrm{PH} 207^{23}$. When necessary, chromosome designations were altered to ensure non-redundant sequence names across parents. Hisat2

247 index files were created using genome sequences only for each contrast. Gene annotations and disjoined filteredTE annotations available at https://github.com/SNAnderson/maizeTE_variation were combined by first subtracting exon regions from the TE annotations and then combining

250 full gene and TE annotations for each genome. Concatenated annotation files were then

251 created for each pairwise contrast using the same chromosomal designation as for the genome

252 files. RNA-seq reads were trimmed using cutadapt ${ }^{34}$ and aligned to the concatenated genomes

253 corresponding to the parents using hisat2 ${ }^{35}$. Unique-mapping reads to the concatenated

254 genome files were then assigned to features (genes and TEs) using HTseq ${ }^{36}$. Counts to each

255 feature were normalized as reads per million using library size estimates derived from the SNP-

256 ASE method (described below). RER for each annotation (gene and TE) was calculated by

257 dividing the mean expression when inherited maternally by the sum of the expression when 258 inherited maternally and paternally. 


\section{Sequence alignments for SNP-ASE}

261 In parallel to the above method of mapping reads, we also ran the standard, SNP-based allele

262 specific expression pipeline by mapping reads to the B73 AGPv4 reference assembly using a 263 variant-aware aligner HiSat2 trained with a set of known SNPs as described in ${ }^{37}$. The number 264 of reads supporting each parental genotype were used to calculate the proportion of maternal 265 reads for each gene. For comparison across mapping methods, genes were filtered for only 266 those with at least 10 informative reads in both methods. SNP-ASE ratios were calculated for 267 each gene in each direction of the reciprocal cross separately by dividing the number of reads matching the maternal allele by the total number of informative reads. Genes with parentspecific expression were defined as those with a SNP maternal ratio $>0.85$ in one direction and $<0.15$ in the reciprocal direction.

\section{Defining imprinting}

273 To define imprinted features using RER, count tables for genes and TEs in each library were

274 loaded into R. For each of the three reciprocal crosses performed in triplicate, DESeq2 ${ }^{38}$ was 275 applied using the IfcThreshold=1 and altHypothesis="greaterAbs" options to identify features 276 with significant deviations from the 2:1 expected expression difference based on dosage. Each 277 contrast includes features from both parental genomes, so maternal and paternal expression 278 was determined by the direction of the differential expression plus the genome where the 279 feature was annotated. Significant features were further filtered to only strong cases of 280 imprinting where RER was $>0.9$ for MEGs and matTEs and $<0.1$ for PEGs. To create the final 281 list of imprinted features, maternal features with pericarp-preferred expression were filtered out 282 (see Tissue Dynamics). 
285 The expression profile of genes and TEs was analyzed for B73 features using previously

286 published analysis ${ }^{29}$ using data from ${ }^{25}$. To filter out genes where expression is higher in the

287 pericarp than the endosperm and could thus result in inaccurate imprinting calls ${ }^{24}$, expression

288 was compared for 14 dap seeds (the time point used in this study) and 18 dap pericarp. Genes

289 with expression over twice as high in the pericarp over the endosperm were excluded from MEG

290 calls. W22 and PH207 genes corresponding to genes expressed higher in the pericarp were

291 also excluded from MEG calls. No matTEs were identified as potential contaminants using this

292 method. Expression data across all tissues was also used to identify endosperm-preferred

293 expression. Endosperm-preferred expression was defined as genes and TEs where the sum of

294 expression in endosperm and wole seed libraries ( $26 \%$ of libraries) was more than $60 \%$ of the

295 sum of expression across all libraries.

\section{Descriptors}

298 To identify genes that are shared between genome assemblies and annotations, the file 299 gene_model_xref_v4.txt was downloaded from MaizeGDB ${ }^{26}$ on 2020/01/22. This file is B73300 based and genes with a single corresponding gene in either the pairwise contrast (for venn 301 diagrams) or in both W22 and PH207 (all other analyses) were defined as conserved in maize 302 while remaining genes were defined as variable. This file was also used to define genes that are 303 syntenic with other grasses, with syntenic genes being defined as any gene with a syntenic 304 ortholog in foxtail millet, rice, brachypodium, and sorghum. To identify the nearest gene to each 305 matTE, bedtools closest was used and distances between TE and gene were reported relative 306 to the orientation of the gene.

308 Data Availability

309 RNA-seq data files have been uploaded to NCBI SRA under BioProject ID PRJNA623806.

310 Scripts and data files used to process results are available at 
bioRxiv preprint doi: https://doi.org/10.1101/2020.04.08.032573; this version posted April 9, 2020. The copyright holder for this preprint (which

was not certified by peer review) is the author/funder, who has granted bioRxiv a license to display the preprint in perpetuity. It is made available under aCC-BY-NC-ND 4.0 International license.

311 https://github.com/SNAnderson/Imprinting2020 and

312 https://github.com/kmhiggins/Imprinting_2020.

313

314 Acknowledgements

315 We thank Peter Hermanson for technical assistance. This work was funded by grants from

316 USDA-NIFA 2016-67013-24747 (SNA, and NMS), NSF IOS-1546899 (PZ and NMS), NSF

317 DGE-1545453 (KH), and from the Minnesota Agricultural Experiment Station (MIN 71-068). The

318 Minnesota Supercomputing Institute (MSI) at the University of Minnesota provided

319 computational resources that contributed to this research.

320 


\section{References}

322 1. Kermicle, J. L. Dependence of the R-mottled aleurone phenotype in maize on mode of sexual transmission. Genetics 66, 69-85 (1970).

2. Hsieh, T.-F. et al. Regulation of imprinted gene expression in Arabidopsis endosperm. Proc. Natl. Acad. Sci. U. S. A. 108, 1755-1762 (2011).

3. Luo, M. et al. A genome-wide survey of imprinted genes in rice seeds reveals imprinting primarily occurs in the endosperm. PLoS Genet. 7, e1002125 (2011).

4. Waters, A. J. et al. Parent-of-origin effects on gene expression and DNA methylation in the maize endosperm. Plant Cell 23, 4221-4233 (2011).

5. Zhang, M. et al. Genome-wide screen of genes imprinted in sorghum endosperm, and the roles of allelic differential cytosine methylation. Plant J. 85, 424-436 (2016).

6. Hatorangan, M. R., Laenen, B., Steige, K. A., Slotte, T. \& Köhler, C. Rapid Evolution of Genomic Imprinting in Two Species of the Brassicaceae. Plant Cell 28, 1815-1827 (2016).

7. Gehring, M., Bubb, K. L. \& Henikoff, S. Extensive demethylation of repetitive elements during seed development underlies gene imprinting. Science 324, 1447-1451 (2009).

8. Ibarra, C. A. et al. Active DNA demethylation in plant companion cells reinforces transposon methylation in gametes. Science 337, 1360-1364 (2012).

9. Park, K. et al. DNA demethylation is initiated in the central cells of Arabidopsis and rice.

Proc. Natl. Acad. Sci. U. S. A. 113, 15138-15143 (2016).

10. Weinhofer, I., Hehenberger, E., Roszak, P., Hennig, L. \& Köhler, C. H3K27me3 profiling of the endosperm implies exclusion of polycomb group protein targeting by DNA methylation. PLoS Genet. 6, (2010).

11. Moreno-Romero, J., Jiang, H., Santos-González, J. \& Köhler, C. Parental epigenetic asymmetry of PRC2-mediated histone modifications in the Arabidopsis endosperm. EMBO J. 35, 1298-1311 (2016).

12. Zhang, M. et al. Genome-wide high resolution parental-specific DNA and histone 
methylation maps uncover patterns of imprinting regulation in maize. Genome Res. 24, 167-176 (2014).

13. Luo, M., Bilodeau, P., Dennis, E. S., Peacock, W. J. \& Chaudhury, A. Expression and parent-of-origin effects for FIS2, MEA, and FIE in the endosperm and embryo of developing Arabidopsis seeds. Proc. Natl. Acad. Sci. U. S. A. 97, 10637-10642 (2000).

14. Waters, A. J. et al. Comprehensive analysis of imprinted genes in maize reveals allelic variation for imprinting and limited conservation with other species. Proc. Natl. Acad. Sci. U.

15. Pignatta, D. et al. Natural epigenetic polymorphisms lead to intraspecific variation in Arabidopsis gene imprinting. Elife 3, e03198 (2014).

16. Wyder, S., Raissig, M. T. \& Grossniklaus, U. Consistent Reanalysis of Genome-wide Imprinting Studies in Plants Using Generalized Linear Models Increases Concordance across Datasets. Sci. Rep. 9, 1320 (2019).

17. Picard, C. L. \& Gehring, M. Identification and Comparison of Imprinted Genes Across Plant Species. Methods Mol. Biol. 2093, 173-201 (2020).

18. Springer, N. M. et al. Maize inbreds exhibit high levels of copy number variation (CNV) and presence/absence variation (PAV) in genome content. PLoS Genet. 5, e1000734 (2009).

19. Hirsch, C. N. et al. Insights into the maize pan-genome and pan-transcriptome. Plant Cell 26, 121-135 (2014).

20. Anderson, S. N. et al. Transposable elements contribute to dynamic genome content in maize. (2019) doi:10.1101/547398.

21. Jiao, Y. et al. Improved maize reference genome with single-molecule technologies. Nature 546, 524-527 (2017).

370 22. Springer, N. M. et al. The maize W22 genome provides a foundation for functional genomics and transposon biology. Nat. Genet. (2018) doi:10.1038/s41588-018-0158-0. 
Genomic and Transcriptome Diversity in Maize. Plant Cell 28, 2700-2714 (2016).

24. Schon, M. A. \& Nodine, M. D. Widespread Contamination of Arabidopsis Embryo and Endosperm Transcriptome Data Sets. Plant Cell 29, 608-617 (2017).

25. Stelpflug, S. C. et al. An Expanded Maize Gene Expression Atlas based on RNA Sequencing and its Use to Explore Root Development. Plant Genome 9, (2016).

26. Portwood, J. L., 2nd et al. MaizeGDB 2018: the maize multi-genome genetics and genomics database. Nucleic Acids Res. 47, D1146-D1154 (2019).

27. Schnable, J. C. Genome evolution in maize: from genomes back to genes. Annu. Rev. Plant Biol. 66, 329-343 (2015).

28. $\mathrm{Xu}, \mathrm{X}$. et al. Resequencing 50 accessions of cultivated and wild rice yields markers for identifying agronomically important genes. Nat. Biotechnol. 30, 105-111 (2011).

29. Anderson, S. N. et al. Dynamic Patterns of Transcript Abundance of Transposable Element Families in Maize. G3 9, 3673-3682 (2019).

30. Batista, R. A. et al. The MADS-box transcription factor PHERES1 controls imprinting in the endosperm by binding to domesticated transposons. Elife 8, (2019).

31. Haig, D. Retroviruses and the placenta. Curr. Biol. 22, R609-13 (2012).

32. Haig, D. Coadaptation and conflict, misconception and muddle, in the evolution of genomic imprinting. Heredity 113, 96-103 (2014).

33. Dilkes, B. P. \& Comai, L. A differential dosage hypothesis for parental effects in seed development. Plant Cell 16, 3174-3180 (2004).

34. Martin, M. Cutadapt removes adapter sequences from high-throughput sequencing reads. EMBnet.journal 17, 10-12 (2011).

35. Kim, D., Langmead, B. \& Salzberg, S. L. HISAT: a fast spliced aligner with low memory requirements. Nat. Methods 12, 357-360 (2015).

36. Anders, S., Pyl, P. T. \& Huber, W. HTSeq--a Python framework to work with highthroughput sequencing data. Bioinformatics 31, 166-169 (2015). 
bioRxiv preprint doi: https://doi.org/10.1101/2020.04.08.032573; this version posted April 9, 2020. The copyright holder for this preprint (which

was not certified by peer review) is the author/funder, who has granted bioRxiv a license to display the preprint in perpetuity. It is made available under aCC-BY-NC-ND 4.0 International license.

399 37. Zhou, P., Hirsch, C. N., Briggs, S. P. \& Springer, N. M. Dynamic Patterns of Gene

400 Expression Additivity and Regulatory Variation throughout Maize Development. Mol. Plant

$401 \quad 12,410-425$ (2019).

402 38. Love, M. I., Huber, W. \& Anders, S. Moderated estimation of fold change and dispersion for

403 RNA-seq data with DESeq2. Genome Biol. 15, 550 (2014). 


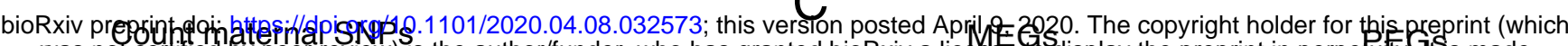
SNP-ASES not Maternal + Paternal available under aCC-BY-NC-ND 4.0 Intermationall license.

B73<smiles></smiles>

NA

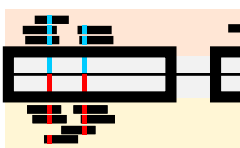

$\mathrm{W} \times \mathrm{B}=1$

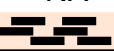

RPM Inherited Maternally Maternally + Paternally

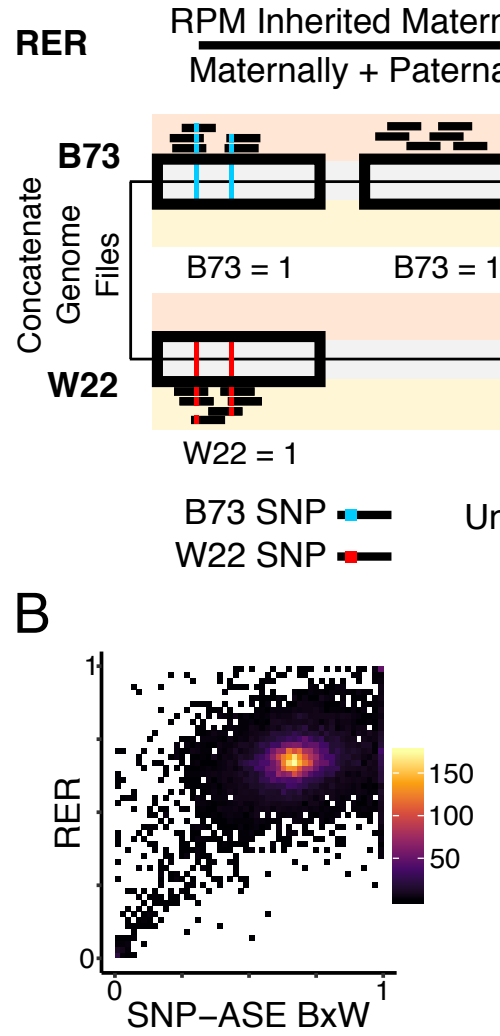

NA Read source

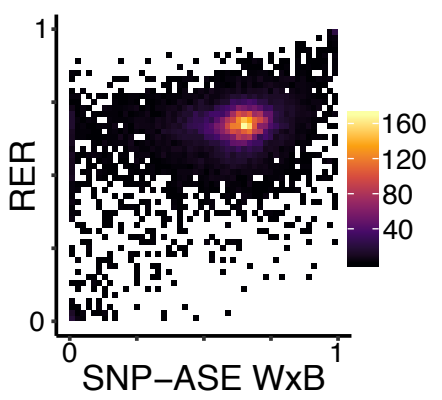

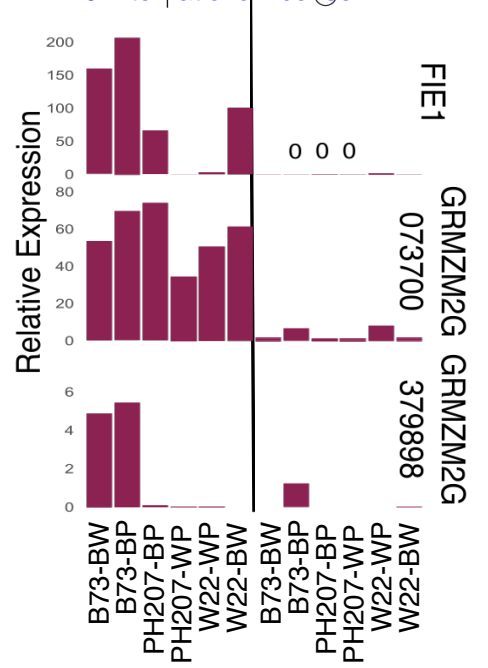

B73 x W22

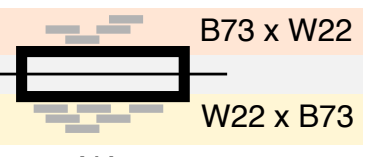

NA
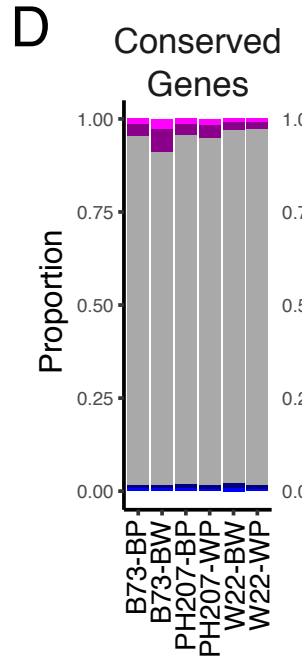
nique Mapping Read Multi Mapping Read $\prod^{\gamma}$

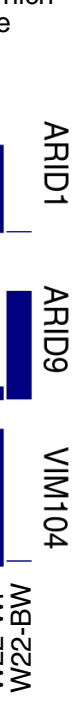

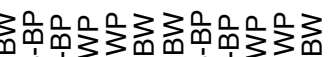

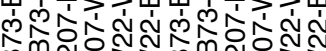

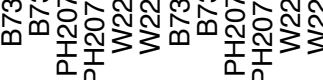

Figure 1: Assessing imprinted expression pattern in maize. A) The method for defining imprinting using SNPASE versus RER. in SNP-ASE, reads mapping to a SNP-corrected reference genome are assigned to alleles based on the SNP supported. In RER, reads are assigned to a concatenated reference genome and retained at unique positions. Both methods can be used to assess imprinting for shared genes distinguished by SNPS, but only RER can assess imprinting for PAV features. B) Comparison of SNP-ASE and RER for B73 genes accessible using both methods in the B73 $\times$ W22 cross, with values plotted showing the average across three biological replicates. SNP-ASE is assessed for each direction of reciprocal crosses separately while RER is calculated with reciprocals. The heat represents the number of genes in each pixel of the plot. C) Expression across all contrasts for genes with conserved imprinting in maize, rice, and Arabidopsis (Waters et al 2011) using the RER method. Bar height represents the mean expression across replicates. Symbols above the plot show whether the gene was inherited maternally or paternally. Gene IDs for these genes are listed in Table S2. D) The distribution of RER values for different features across contrasts. RER cutoffs for strong maternal and strong paternal are $>0.9$ and $<0.1$, respectively, and cutoffs for moderate maternal and paternal are $>$ 0.8 or $<0.2$, respectively. 
bioRxiv preprint doi: https://doi.org/10.1101/2020.04.08.032573; this version posted April 9, 2020. The copyright holder for this preprint (which Figure Rt certified by peer review) is the author/funder, who has granted bioRxiv a license to display the preprint in perpetuity. It is made available under aCC-BY-NC-ND 4.0 International license.
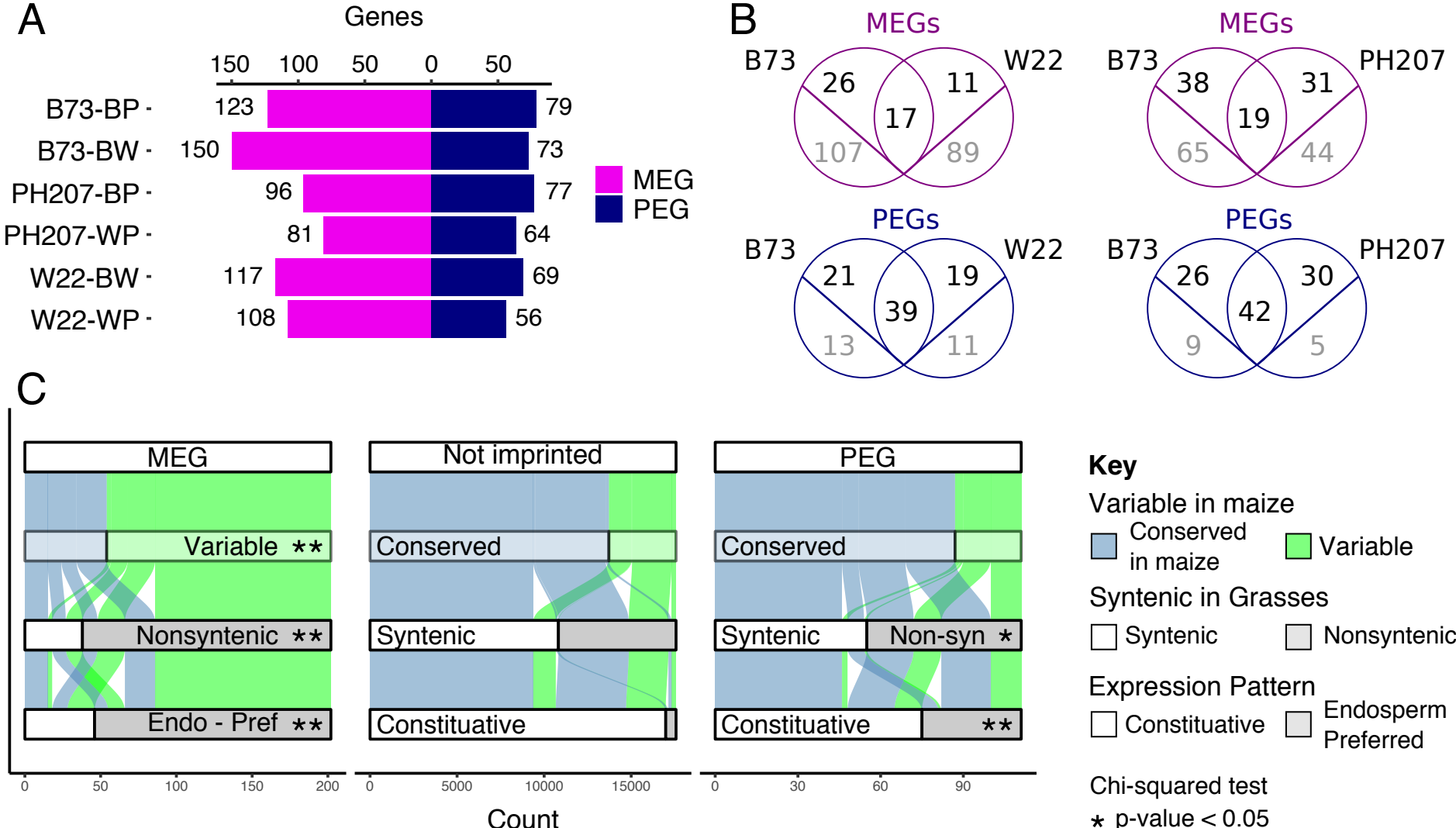

\section{Key}

Variable in maize
$\square$ Conserved in maize
$\square$ Variable

Syntenic in Grasses
$\square$ Syntenic $\square$ Nonsyntenic
Expression Pattern
$\square$ Constituative Endosperm

Chi-squared test

$\star \mathrm{p}$-value $<0.05$

$\star *$-value $<0.001$

Figure 2: Imprinting of genes defined by RER. A) The number of imprinted genes identified across contrasts using the RER method (see methods). MEGs are shown in magenta and PEGs are shown in blue. B) The overlap between imprinted genes across pairwise contrasts. Genes that are shared between genotypes that could be assessed for imprinting are shown in black above the line while imprinted genes unique to one genome are shown in gray below the line. C) Comparison of features for MEGs, PEGs, and non-imprinted B73 genes. Genes are defined as conserved when they are shared with all genotypes present in this study, syntenic when a syntenic ortholog exists in sorghum, rice, foxtail millet, and brachypodium, and endospermpreferred if expression is primarily restricted to the endosperm (Figure S4). Asterisks denote significance relative to the Not Imprinted set (chi-squared test). 
bioRxiv preprint doi: https://doi.org/10.1101/2020.04.08.032573; this version posted April 9, 2020. The copyright holder for this preprint (which was not certified by peer review) is the author/funder, who has granted bioRxiv a license to display the preprint in perpetuity. It is made available under aCC-BY-NC-ND 4.0 International license.

\section{Figure 3}

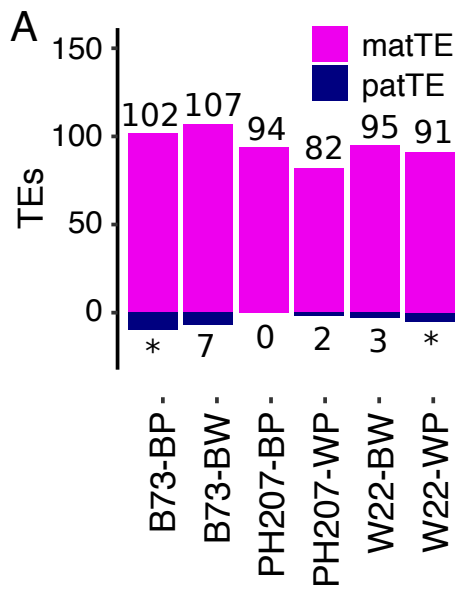

D MEG PEG

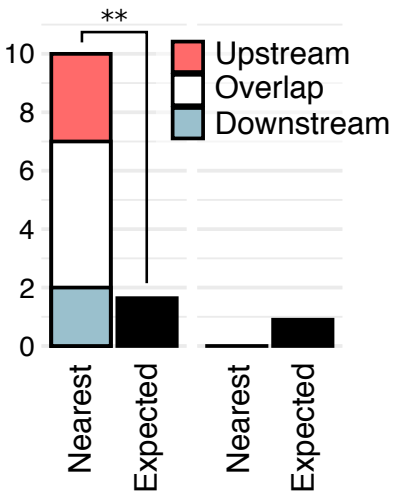

B

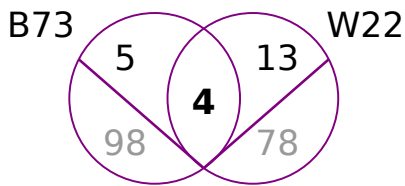

B73

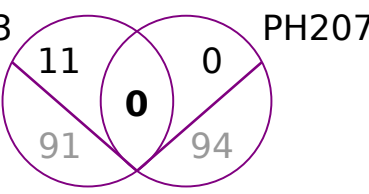

C

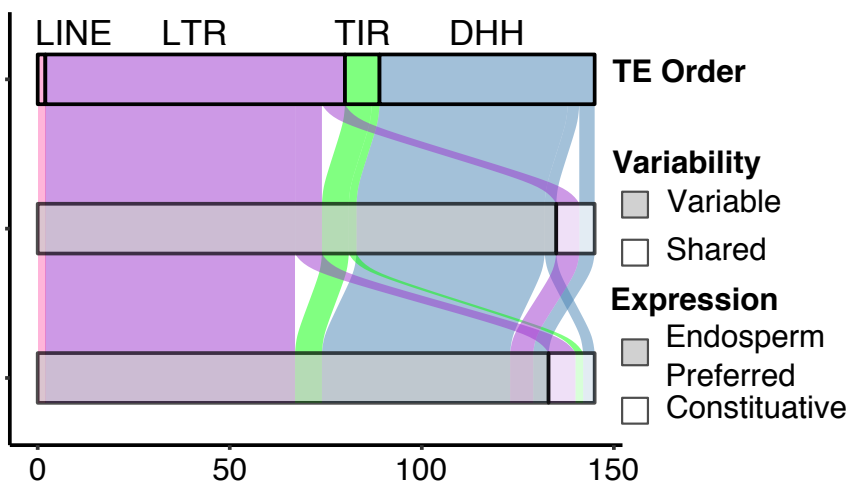

$E$

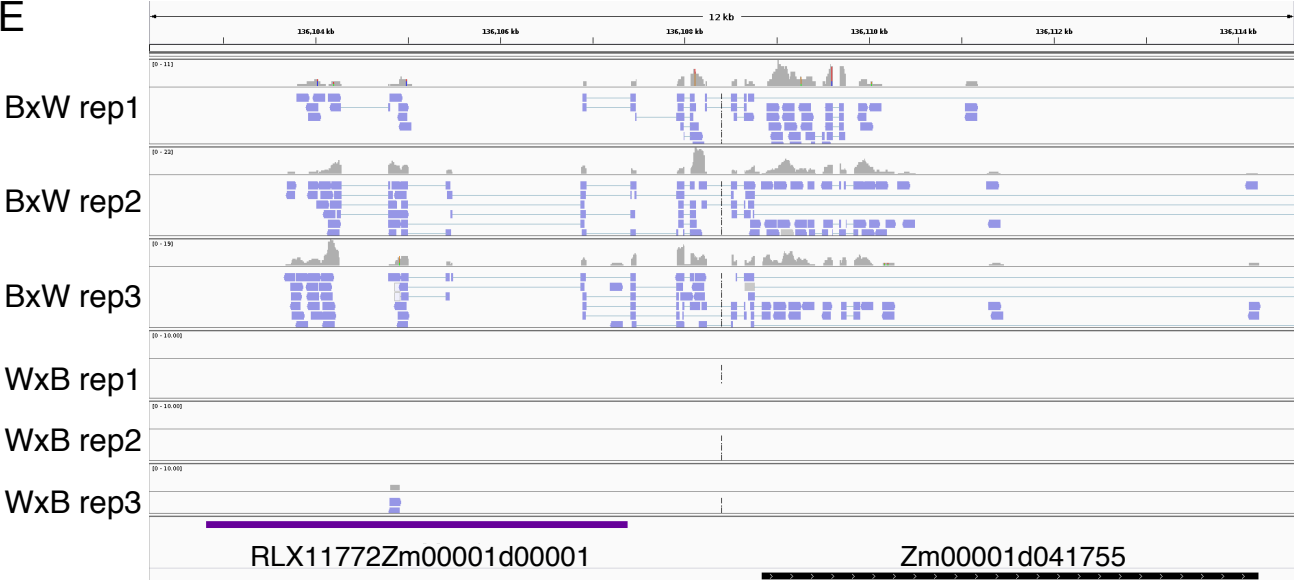

Figure 3: Imprinted TEs defined by RER. A) The number of imprinted TEs across contrasts. matTEs are marked in magenta and paternally expressed TEs are marked in navy. Asterisks denote contrasts where paternally expressed TEs could not be defined (Figure S2). B) The overlap between matTEs across pairwise contrasts. TEs that are shared between genotypes that could be assessed for imprinting are shown in black above the line while imprinted TEs unique to one genome are shown in gray below the line. C. Features of matTEs in B73. TE orders are abbreviated: $\mathrm{DHH}=$ Helitron, $\mathrm{TIR}=$ terminal inverted repeat transposon, LTR $=$ LTR retrotransposon, and LINE = long interspersed nuclear element. TE variability is defined by prior work (Anderson et al. 2019). Endosperm-preferred expression is described by patterns across development (Figure S4). D) Imprinting status of closest gene to matTEs. Expected number is based on the number of MEGs and PEGs that were assessed for imprinting. ${ }^{* *} p$-value $<0.001$ (binomial test) E) IGV view showing a representative example of reads aligning to a matTE near a MEG. Reads are colored by the strand of alignments, where blue = forward strand. 